Heylen, E., Deconinck, G., Van Hertem, D. (2016). Impact of increased uncertainty in power systems on performance of short term reliability management.

This is the author's version of an article that has been accepted for publication in Conference proceedings of International conference on Probabilistic methods applied to power systems 2016 (PMAPS 2016). Changes are made to this version by the publisher prior to publication.

(C) 2016 IEEE. Personal use of this material is permitted. Permission from IEEE must be obtained for all other users, including reprinting/ republishing this material for advertising or promotional purposes, creating new collective works for resale or redistribution to servers or lists, or reuse of any copyrighted components of this work in other works. 


\title{
Impact of Increased Uncertainty in Power Systems on Performance of Short Term Reliability Management
}

\author{
Evelyn Heylen, Geert Deconinck, Dirk Van Hertem \\ Department of Electrical Engineering, Div. Electa \\ KU Leuven, EnergyVille \\ Leuven, Belgium \\ Email: evelyn.heylen@esat.kuleuven.be
}

\begin{abstract}
Power system states are not accurately known ahead of real time and are subject to natural variability, which leads to uncertainty in the system. Sources of uncertainty are amongst others load, renewable energy sources and contingencies. An aggregate measure for system's uncertainty is determined in order to have a single quantified value of the uncertainty level in the system. This allows to compare performance of power system reliability management according to various reliability criteria at different uncertainty levels in a transparent manner. A case study for a 5 node test system illustrates the use of the aggregate uncertainty measure and the impact of different uncertainty levels on short term reliability management of transmission system operators according to three reliability criteria. Results of the case study illustrate that probabilistic reliability management can lead to significant improvements in performance in terms of total system cost and curtailment of non-flexible load compared to reliability management based on deterministic $\mathrm{N}-\mathrm{0}$ and $\mathrm{N}-1$ criteria, especially at high uncertainty levels in heavily loaded system conditions.

Index Terms-Power system operation, power system reliabil-
\end{abstract} ity, reliability criterion, reliability management, uncertainty.

\section{INTRODUCTION}

Uncertainty can be classified in stochastic uncertainty and epistemic/knowledge-based uncertainty. The main difference between them is that knowledge-based uncertainty can be reduced in contrast to stochastic uncertainty, which is by definition irreducible. Three major groups of uncertainty can be defined [1]:

- Model uncertainty: The applied model is a simplification of reality.

- Completeness uncertainty: Not all contributions to the risk are/can be addressed. For instance, it is infeasible to assess all possible system states in large systems.

- Variables' uncertainty: Variables in the model might be not accurately known, subject to natural variability and stochastic in nature, such as net load and wind generation.

The aim of this paper is to assess the impact of stochastic variables' uncertainty on performance of short term power system reliability management of a transmission system operator (TSO) according to various reliability criteria.

The work of Evelyn Heylen is supported by the Research Foundation Flanders (FWO)

The research leading to these results has received funding from the European Union Seventh Framework Programme under Grant Agreement No 608540 .
Power systems are one of the most critical infrastructures for modern society and require an adequate reliability level. Nowadays, power system reliability is managed according to a deterministic N-1 criterion, which has various shortcomings regarding the management of uncertainty, such as the fact that it considers only $\mathrm{N}-1$ contingencies with equal probability and severity and no forecast errors for load and wind power generation. Probabilistic reliability criteria consider uncertainties in a more convenient way and can overcome shortcomings of deterministic criteria.

The preventive decision stage of the short term decision making process of a TSO is prone to uncertainty about real time system states, such as real time realizations of load and generation of renewable energy sources and possible contingencies, i.e. unexpected failures or outages of a system component, such as a generator, transmission line, circuit breaker, switch, or other electrical element [2]. Different reliability criteria cause operators to treat this uncertainty in different ways. Performance of reliability criteria depends on the uncertainty level in the system, which is determined by the accuracy of load and Renewable Energy Source (RES) forecasts and possible contingencies in the system, and how well the system is prepared for the uncertainties, which results from reliability management. In order to give incentives to power system stakeholders to change their manner of reliability management towards a more cost effective approach, it is crucial to quantify changes in socio-economic and reliability performance of using more complex and more computationally intensive probabilistic reliability approaches [3], [4].

Studies have illustrated the impact of load uncertainty on system reliability indices and bus reliability indices [5]-[7]. Reliability management according to various reliability criteria is not considered in these studies. Uncertainty is mostly defined in terms of correlation matrices and standard deviations in literature, assuming normal distributions of uncertain variables. In order to facilitate the analysis and comparison of cases with similar orientation of the probability pattern of the uncertain variables, an overall measure of uncertainty is proposed quantifying system's uncertainty level in a single value. Section II describes the aggregate measure of uncertainty. Section III gives an overview of the methodology used to assess the impact of uncertainty on performance of various 
reliability criteria in short term reliability management. In section IV, the methodology is applied to a 5 node test system. Section V discusses results of the case study, while section VI summarizes the key take-aways.

\section{Measure of AGgRegate System UnCERTAinty}

Uncertain variables in power systems are for instance wind generation, load and contingencies. Moreover, reliability data such as failure and repair rates of system components might be not precise due to the small number of events occurring. Uncertainty is often dealt with by assigning probability distributions to the variables. Next to the uncertainty of those variables, values are correlated in time and in space. Spatial correlation exists not only between variables of the same type, for example between the output of wind generators at different locations [8], but also between values of variables of different types such as for instance between wind generation and load [9]. Uncertainty and correlation of different variables are typically expressed in a covariance matrix. The covariance matrix captures the orientation and size of the uncertainty cloud [10]. If the uncertainty cloud is a multi-variate normal distribution, the covariance matrix is the parameter of variance of the distribution and both upward and downward deviations of the forecast values are possible. However, in reality, if a wind generator hits its maximal generation capacity, only downward deviations are possible, resulting in non-symmetric distributions. This is not considered in the presented study.

In order to easily compare results in terms of uncertainty, an aggregate measure of uncertainty, representing the dispersion of all variables together in a single number, is determined. Two overall measures of dispersion are total variation and generalized variance [11]. Total variation (TV) is defined as the sum of all variances along the different components in the system or the trace of the covariance matrix cov.

$$
T V=\operatorname{trace}(\operatorname{cov})
$$

A problem with total variation as a measure of dispersion is that it does not take into account any information about the correlation among variables in contrast to generalized variance [11]. Generalized variance $(\mathrm{GV})$ equals the product of eigenvalues or the determinant of the covariance matrix [12].

$$
G V=\operatorname{det}(\operatorname{cov})=\prod_{k} \lambda_{k}
$$

The generalized variance can be considered as representative for the total uncertainty in the system. Generalized variance as aggregate uncertainty measure can be explained based on principal component analysis (PCA) of the covariance matrix of system variables, such as load and renewable generation. Principal component analysis explains the variance-covariance structure of a set of variables using linear combinations of the variables. It consists of making an eigenvalue decomposition of the covariance matrix. Eigenvectors of the covariance matrix represent the principal components of the system, which are independent directions with maximum variability. The variance along a particular direction is given by the

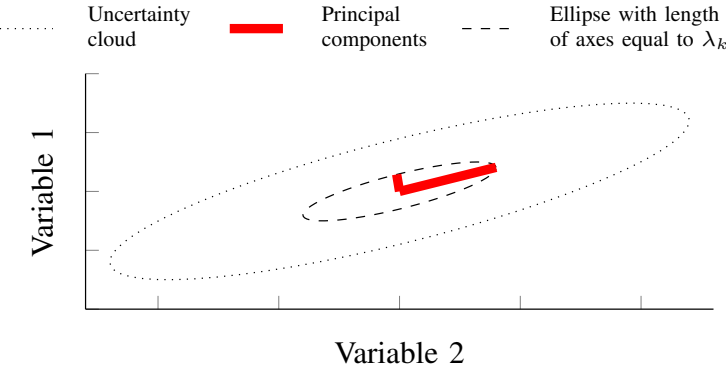

Fig. 1. Two dimensional illustration of the proportionality between the aggregate measure of uncertainty based on generalized variance and the surface area of the uncertainty cloud

eigenvalue $\lambda_{k}$ corresponding to the eigenvector of the direction under analysis [12]. The distributions they are representing are independent. The eigenvalues of the covariance matrix are proportional to the half-length of the principal axes of the higher dimensional ellipsoid approximating the uncertainty cloud of uncertain system variables. As GV is the product of the eigenvalues of the covariance matrix, it is proportional to the product of the half lengths of the higher dimensional ellipsoid. Therefore, the generalized variance is proportional to the higher dimensional volume of the ellipsoid that can be imagined to represent the uncertainty cloud. This is graphically illustrated in Fig. 1 for a two dimensional case.

Generalized variance does not contain any information on the orientation of the patterns [12]. Therefore, generalized variance is easier to interpret when the samples being compared have nearly the same orientation. In the considered analysis, the orientation of the uncertainty cloud, representing uncertainty in RES and load, is similar for different cases.

The generalized variance is dependent upon the scale of the variables in the sample [13]. All uncertain variables forming the uncertainty cloud have equal units in this analysis. In order to be able to compare simulations in an explicit way, uncertainty $U=\sqrt[n]{G V}$ is used, which corresponds to the length of the side of a n-dimensional cube of which the volume equals the generalized variance.

\section{Methodology}

In order to assess the impact of uncertainty on the performance of short term reliability management, the TSO decision making process according to various reliability criteria needs to be simulated for different uncertainty levels. An overview of the simulation procedure is given in Fig. 2. The procedure consists of four parts:

- Generation of a sample of system states to assess, representing the uncertainty in the system

- Simulation of short term reliability management

- Evaluation of reliability decision related trajectory and final system states in the sample

- Post-processing of the results

The coming subsections elaborate more on each of the parts. 


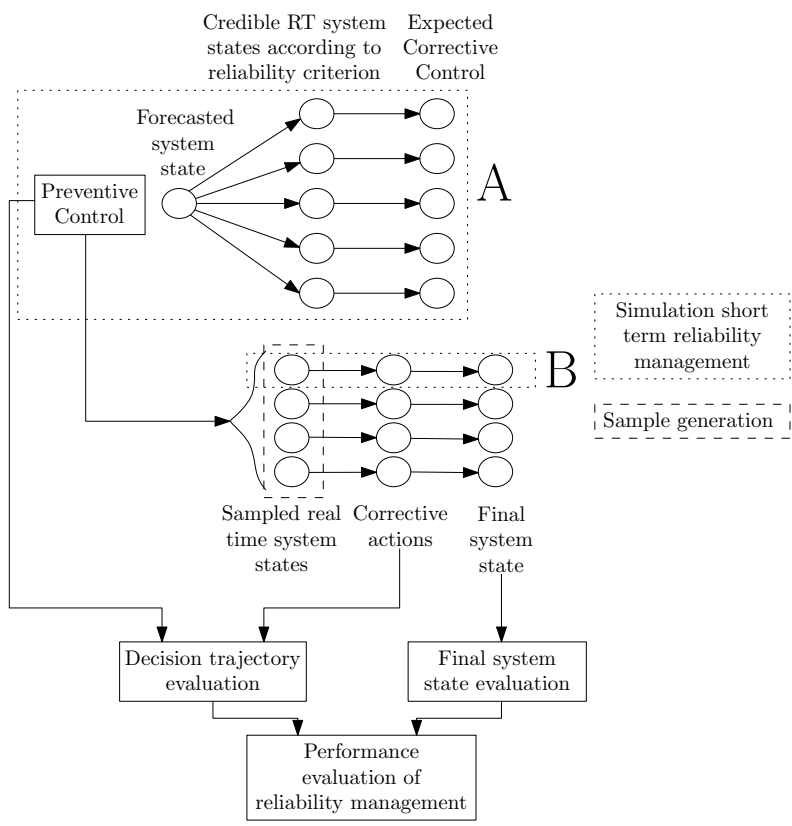

Fig. 2. Overview of the simulation procedure

\section{A. Simulation of TSO's short term reliability management}

TSO's short term decision making process consists of operational planning (OP) and real time operation (RT). The simulation of the TSO decision making process of short term reliability management is indicated by the dotted boxes in Fig 2. Initially, preventive control actions for a particular expected state are determined taking into account the reliability criterion. This is shown in part A of the simulation of short term reliability management in Fig 2. Constraints and credible real time system states considered in the operational planning decision stage differ between reliability criteria, resulting in different preventive control actions that are selected.

Real time system states differ from credible system states considered in the preventive decision stage due to uncertainties in the system. The real time decision making process is simulated resulting in corrective actions. Preventive actions are taken into account in the simulation of corrective control. Applying corrective actions in real time will result in a final system state. This process is shown in part B of the simulation of short term reliability management in Fig. 2.

\section{B. Sample generation}

In theory, all possible system states should be evaluated in order to make an assessment of the performance of reliability management according to a particular reliability criterion that completely captures the impact of uncertainty. However, this is not doable in practice, especially not in large systems. A sampling procedure determines system states to evaluate in the corrective control stage of short term reliability management, as indicated by the dashed box in Fig. 2. System states in the sample represent possible realizations of uncertain system variables, such as load and wind power generation. Latin Hypercube Sampling (LHS) [14] is used to generate samples representing different uncertainty levels. LHS requires that variables are independent [15]. Principal component analysis (PCA) is used in order to generate independent, normal distributions approximating the multi-dimensional, correlated probability structure of the uncertainty cloud. 1000 real time system states are generated per sample related to a particular set of forecast values. All system states in the sample have equal probability of occurring as the probability structure is taken into account in the sampling procedure.

Uncertainty due to contingencies is included using an analytical approach. Component reliability data, such as failure and repair rates, are used to determine the probability of a particular contingency. For each real time system state of load and RES generation in the sample, the most probable contingencies up to a particular cumulative probability are simulated. Outcomes according to different contingencies are weighted using the probability of the contingencies.

\section{Performance evaluation}

Performance of power system reliability criteria is expressed in terms of expected total system cost (TC). Expected total system cost per real time system state $s$ determined by a particular load and RES power realization consists of the cost of actions and the interruption cost and is defined as:

$$
T C=C_{\text {prev }}+\sum_{c=1}^{C} p_{c} \cdot\left[C_{\text {corr }, c}+\sum_{j=1}^{L} C_{c u r t, j, c}\right]
$$

with $C_{\text {prev }}$ the cost of preventive actions, $C_{c o r r, c}$ the cost of corrective actions in contingency case $c, C_{c u r t, j, c}$ the cost of load curtailment at load point $j$ in contingency case $c$ and $p_{c}$ the probability of contingency $c$. In this way, both the final system state and the reliability decision related trajectory are evaluated, which is required to make a complete evaluation of the performance of reliability management according to a particular reliability criterion. Expected total system cost is evaluated for all system states in the sample. Overall expected total cost can be calculated as:

$$
E T C=p_{s} \cdot T C
$$

with $p_{s}$ the probability of system state $s$ in the sample. $p_{s}$ is the same for all system states in the sample in the used sampling procedure and equals $\frac{1}{N}$ with $N$ the number of system states in the sample.

\section{Post-processing}

Post-processing focusses on two different kinds of information. On the one hand a trimmed data set is used omitting the $6.5 \%$ highest and lowest outcomes in terms of $T C$ in order to omit outliers. The trimmed data set is analysed using the trimmed mean combined with the interquartile distance of the trimmed data set in order to analyse the spread of the data. On the other hand, the number of cases in which non-flexible load is curtailed is investigated. These cases are denoted as Load Curtailment Cases (LCCs) in the remainder of the paper. 
As only samples are investigated in the analysis, significance tests are used that give evidence whether an observed effect in the sample reflects an effect that is present in the population. Moreover, they are useful to compare different samples [16]. Due to outliers and the asymmetric probability density function of the data, traditional t-tests cannot be used as the assumptions of these tests are not satisfied. Non-parametric permutation tests are used. The normality condition does not need to be satisfied for permutation tests and they are robust in the sense that outcomes are still accurate even when the two population distributions under investigation have slightly different standard deviations [16]. Permutation tests use resample methods without replacement to generate samples of the performance indicators that represent the null-hypothesis. In order to determine the significance of differences in mean total system cost for different reliability criteria at a particular uncertainty level, a significance test for paired data is required, because the same system states are evaluated for different reliability criteria at a particular uncertainty level. Paired and unpaired tests can be distinguished based on the difference in permutation resampling method of the sample of result data. The hypotheses are:

$$
\begin{aligned}
& H_{0}: \overline{T C} C_{\text {rrit }_{1}}-\overline{T C} C_{\text {rrit }_{2}}=0 \\
& H_{1}: \overline{T C} C_{\text {rrit }_{1}}-\overline{T C} C_{\text {rrit }_{2}}>0
\end{aligned}
$$

with $\overline{T C} C_{\text {crit }_{i}}$ the mean total system cost according to a particular reliability criterion $i$. An $\alpha$-value of 0.05 is used.

\section{CAse STUdy}

The impact of uncertainty in power systems on performance of short term reliability management based on different reliability criteria is analysed for a case study on a 5 node test system. Short term reliability management based on three different reliability criteria is simulated for various levels of aggregate uncertainty.

\section{A. Test system}

The 5 node test system based on the Roy Billinton Reliability test system (RBTS) is shown in Fig. 3 [17], [18]. Three

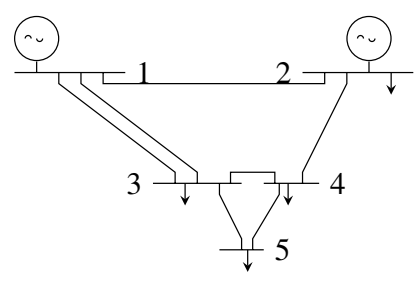

Fig. 3. The five node test system

types of uncertainty are taken into account: contingencies, wind generation and load. Components are considered to have constant failure rates and repair times. The impact of imprecise reliability data is not considered in this case study.

Information about the generators and average spatial load distribution in the test system is summarized in table I. Wind
TABLE I

OVERVIEW OF GENERATORS AND AVERAGE SPATIAL LOAD DISTRIBUTION IN THE TEST SYSTEM

\begin{tabular}{l|cc||lc}
\hline Node & $\begin{array}{l}\text { Generation } \\
\text { capacity [MW] }\end{array}$ & Type & Node & $\begin{array}{c}\text { Average } \\
\text { Load share [\%] }\end{array}$ \\
\hline 1 & 40 & Conventional & 1 & 0 \\
1 & 40 & Conventional & 2 & 12.12 \\
1 & 50 & Wind & 3 & 51.52 \\
2 & 40 & Conventional & 4 & 24.24 \\
2 & 20 & Conventional & 5 & 12.12 \\
2 & 100 & Wind & & \\
2 & 5 & Conventional & & \\
2 & 5 & Conventional & & \\
\hline
\end{tabular}

generators represent $50 \%$ of total generation capacity in the system, however, the output of those wind generators is not certain. Different uncertainty levels are investigated, leading to different generalized variances and uncertainties $U$ for two load levels, as summarized in table II. $20 \%$ of total system load is available for curtailment at a cost, while the remainder of the load can only be curtailed in emergency cases.

TABLE II

OVERVIEW OF CONSIDERED COMBINATIONS OF LOAD AND WIND GENERATION FORECASTS AND CONSIDERED UNCERTAINTY LEVELS

\begin{tabular}{l|ccccc}
\hline $\begin{array}{l}\text { Case } \\
\text { no. }\end{array}$ & $\begin{array}{c}\text { Total load } \\
\text { forecast [MW] }\end{array}$ & $\begin{array}{c}\text { Total wind } \\
\text { forecast [MW] }\end{array}$ & $\begin{array}{c}U_{1} \\
{[\mathrm{MW}]}\end{array}$ & $\begin{array}{c}U_{2} \\
{[\mathrm{MW}]}\end{array}$ & $\begin{array}{c}U_{3} \\
{[\mathrm{MW}]}\end{array}$ \\
\hline $\mathrm{C} 1$ & 135 & 110 & 1.2 & 2.2 & 3.2 \\
$\mathrm{C} 2$ & 175 & 110 & 1.7 & 3.1 & 4.6 \\
\hline
\end{tabular}

(Cross-)correlation between wind generation and load at different nodes in the system is taken into account. The correlation matrix used in this study, which is the normalized version of the covariance matrix, is shown in (5) [8], [9].

$$
\text { Corr }=\left[\begin{array}{cccccc}
1 & 0.95 & 0.047 & 0.064 & 0.03 & 0.025 \\
0.95 & 1 & 0.07 & 0.04 & 0.03 & 0.01 \\
0.047 & 0.07 & 1 & 0.2 & 0.35 & 0.15 \\
0.064 & 0.04 & 0.2 & 1 & 0.22 & 0.3 \\
0.03 & 0.03 & 0.35 & 0.22 & 1 & 0.3 \\
0.025 & 0.01 & 0.15 & 0.3 & 0.3 & 1
\end{array}\right]
$$

\section{B. Reliability management}

The focus of the paper is on TSOs' short term reliability management, consisting of operational planning and real time operation. Generation redispatch is available as preventive action in the operational planning stage. Operational planning decision making also takes into account available actions in real time. Considered real time corrective actions are generation redispatch and load curtailment [4]. Reliability is managed according to three reliability criteria:

- N-0 criterion: No load curtailment or violation of operational limits allowed in the $\mathrm{N}-0$ system state for the forecast value of load and wind generation.

- N-1 criterion: No load curtailment or violation of operational limits allowed in all contingency cases up to 
$\mathrm{N}-1$ system states for the forecast value of load and wind generation. All contingencies are assumed to be equally probable. Only branch outages are considered.

- Probabilistic reliability criterion: The objective is to minimize expected total system cost. Load uncertainty is taken into account in probabilistic reliability management ahead of real time using load scenarios. These load scenarios are derived from a discretized normal distribution with the load forecast as mean $\mu$ and a standard deviation $\sigma$. A similar approach is used to take into account uncertainty of renewable generation. The load and RES scenarios range from $\mu-3 \sigma$ up to $\mu+3 \sigma$, with equal spacing between the scenarios. Moreover, contingency cases up to a cumulative probability of $99 \%$ are considered. All system states are taken into account with their respective probability of occurring [4]. For the 5 node test system, 5 wind generation scenarios and 7 load scenarios are considered in the operational planning stage ahead of real time, together with the contingency cases. This results in 280 considered system states for the 5 node test system. The standard deviations of the distributions representing possible load and wind generation realizations given a particular forecast equal $4 \%$ and $6 \%$ respectively.

The TSO decision making process is simulated using a DC SCOPF based on MATPOWER's extensible OPF formulation [4], [19]. Performance of reliability management is evaluated based on the same system states for the three reliability criteria. Contingency cases up to a cumulative probability of 99.99\% are assessed for all system states generated by the Latin Hypercube Sampling technique as discussed in section III-B for the cases summarized in table II.

\section{RESULTS}

Probability distributions of total system cost $T C$ obtained from the samples are not normal, but right-skewed with outliers. Table III gives the expected total system cost ETC expressed relatively to the expected total system cost if the $\mathrm{N}$ 0 reliability criterion is applied in the high $(\mathrm{C} 2)$ and low load (C1) case for the three uncertainty levels specified in table II. The permutation significance test shows that differences in mean total system cost between the reliability criteria are significant for the three uncertainty levels. The application of a N-1 reliability criterion reduces expected total system cost, especially at high uncertainty levels. Cost savings are even larger if a probabilistic reliability criterion is applied, especially if the system is more heavily loaded. The focus in the remainder of this section will be on the results of the heavily loaded case $\mathrm{C} 2$.

Fig. 4 gives the number of load curtailment cases (LCCs) that occur in case $\mathrm{C} 2$ if different reliability criteria are applied, expressed relatively to the number of load curtailment cases if the $\mathrm{N}-0$ criterion is applied in the case with uncertainty level $U_{3}$. The number of load curtailment cases can be reduced if a $\mathrm{N}-1$ or probabilistic reliability criterion is used, with the largest difference at the highest uncertainty level. N-1 and probabilistic reliability criteria lead to a similar number of
TABLE III

EXPECTED TOTAL SYSTEM COST ETC EXPRESSED RELATIVELY TO THE ETC IF THE N-0 CRITERION IS APPLIED IN CASE C1 AND C2 OF TABLE II

\begin{tabular}{l|l|ccc}
\hline Case & Criterion & $U_{1}$ & $U_{2}$ & $U_{3}$ \\
\hline \multirow{3}{*}{$\mathrm{C} 1$} & N-0 & 100 & 100 & 100 \\
& N-1 & 99.8 & 99.8 & 97.2 \\
& Probabilistic & 97.1 & 96.0 & 93.6 \\
\hline \multirow{3}{*}{ C2 } & N-0 & 100 & 100 & 100 \\
& N-1 & 95.0 & 91.9 & 74.9 \\
& Probabilistic & 92.4 & 89.8 & 72.9 \\
\hline
\end{tabular}

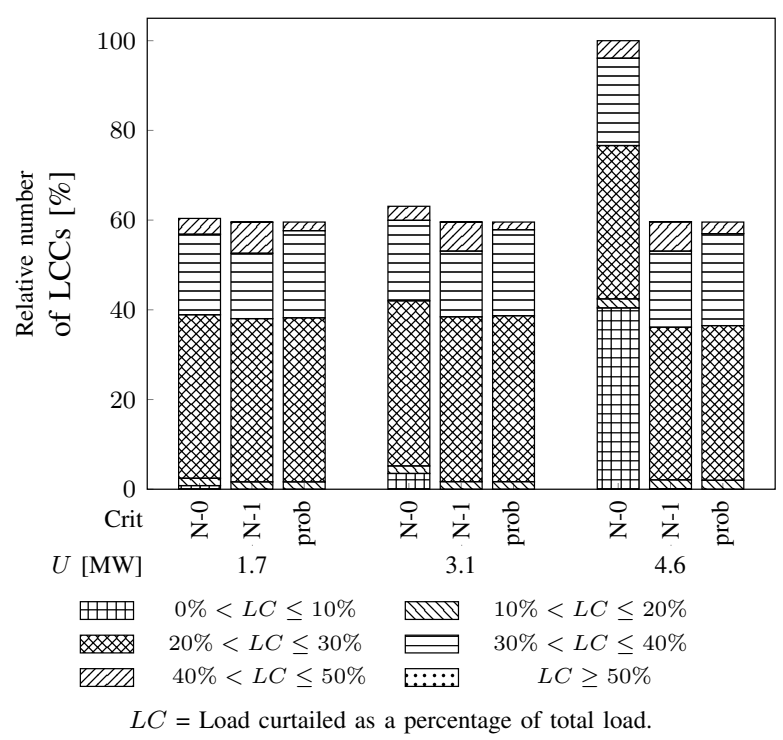

Fig. 4. Relative number and severity of load curtailment cases (LCCs) in the heavily loaded case $\mathrm{C} 2$ for three reliability criteria for the three uncertainty levels specified in table II.

load curtailment cases, however, the probabilistic reliability criterion results in a lower amount of load curtailed in part of the cases, as illustrated by the stacks in Fig. 4, which represent the number of LCCs with an amount of load curtailed (LC) within the specified range expressed as a percentage of total load.

Fig. 5 gives the $6.5 \%$ trimmed mean total system cost $T C$ and the $25 \%$ and $75 \%$ percentiles of the trimmed data for case C2 for different levels of aggregate uncertainty. Results are expressed relatively to the maximal trimmed mean total system cost, i.e. if the N-0 reliability criterion is applied at the highest uncertainty level. The trimmed mean total system cost is lower with a $\mathrm{N}-1$ criterion compared to a $\mathrm{N}-0$ criterion at each uncertainty level. The probabilistic reliability criterion leads to even lower total system costs. Also the interquartile distance is smaller if the probabilistic reliability criterion is applied. The $25 \%$-percentile of the trimmed total system cost sample is nearly constant as a function of the uncertainty level for all three reliability criteria, but the trimmed mean and $75 \%$-percentile increase, leading to an increased spread 


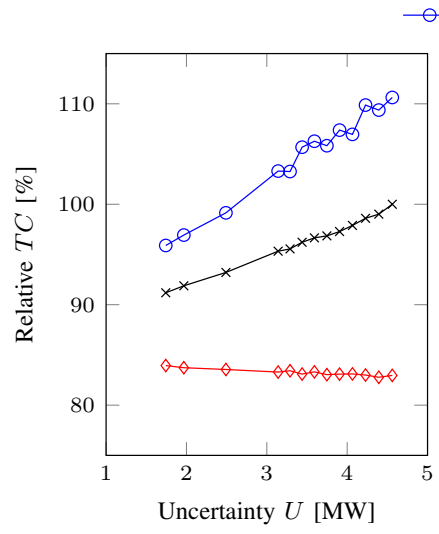

(a) $\mathrm{N}-0$ criterion

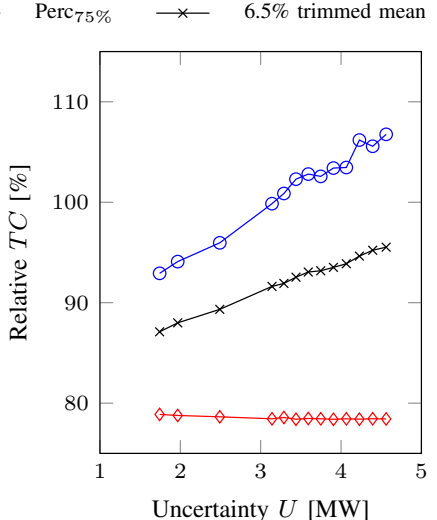

(b) N-1 criterion

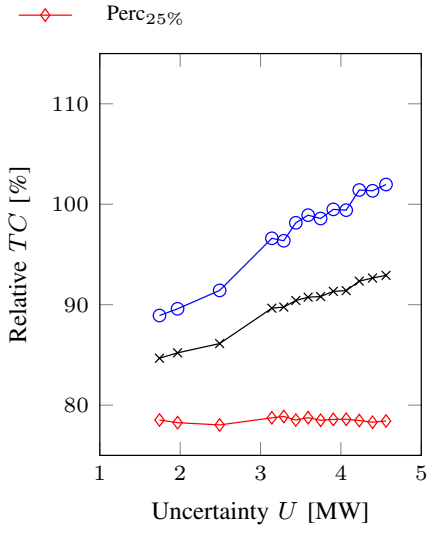

(c) Probabilistic criterion

Fig. 5. Evolution of the mean and spread of the $6.5 \%$ trimmed data samples of total system cost $T C$ as a function of the uncertainty level $U$ for the three reliability criteria under investigation in the heavily loaded 5 node test system (C2). Values of $T C$ are expressed relatively to the maximal trimmed mean $T C$, i.e. if the $\mathrm{N}-0$ criterion is applied.

of total system cost at higher uncertainty levels.

\section{CONCLUSION}

Probabilistic reliability criteria for short term power system reliability management consider uncertainty related to load and RES forecasts and contingencies in a more convenient way compared to deterministic reliability criteria. A single, aggregate measure of uncertainty based on generalized variance allows to make a transparent comparison of performance of power system reliability management according to various reliability criteria in terms of level of uncertainty in the system. A case study on a 5 node test system shows potential improvements in terms of socio-economic performance and curtailment of non-flexible load if a probabilistic reliability criterion is applied. Expected total system cost is lower and also the spread on total system cost is reduced if a probabilistic criterion is used. The effect is even more pronounced in heavily loaded cases with high uncertainty levels.

The proposed aggregate uncertainty measure can be used in a sequential assessment in future work, which allows to assess the impact of temporal effects. Moreover, the impact of spatial and temporal correlation and imprecise reliability data on the performance of reliability management can be assessed.

\section{REFERENCES}

[1] M. Abrahamsson, "Uncertainty in quantitative risk analysis - characterisation and methods of treatment," Report 1024, Department of Fire Safety Engineering, Lund University, Lund, 2002.

[2] Garpur consortium, "State of the art on reliability assessment in power systems," [Online]: http://www.garpur-project.eu/deliverables, 2014.

[3] E. Heylen and D. Van Hertem, "Importance and difficulties of comparing reliability criteria and the assessment of reliability," in Young Researchers Symposium, 2014. IEEE, 2014.

[4] E. Heylen, W. Labeeuw, G. Deconinck, and D. Van Hertem, "Framework for evaluating and comparing performance of power system reliability criteria," Power Systems, IEEE Transactions on, 2016 (In press).

[5] W. Li and R. Billinton, "Effect of bus load uncertainty and correlation in composite system adequacy evaluation," Power Systems, IEEE Transactions on, vol. 6, no. 4, pp. 1522-1529, 1991.
[6] R. Billinton and R. N. Allan, "Power system reliability in perspective," Electronics and Power, vol. 30, no. 3, pp. 231-236, Mar. 1984.

[7] D. Zhai, A. Breipohl, F. Lee, and R. Adapa, "The effect of load uncertainty on unit commitment risk," Power Systems, IEEE Transactions on, vol. 9, no. 1, pp. 510-517, 1994.

[8] E. Nuno Martinez, N. A. Cutululis, and P. Sørensen, "Future wind generation scenarios," Presentation GARPUR workshop, 2015.

[9] R. Billinton, R. Karki, and A. K. Verma, Reliability and risk evaluation of wind integrated power systems. Springer, 2013.

[10] H. E. A. Tinsley and S. D. Brown, Handbook of applied multivariate statistics and mathematical modeling. Academic Press, 2000.

[11] "Additional measures of dispersion," [Online] Available: https:// onlinecourses.science.psu.edu/stat505, 2016.

[12] R. A. Johnson and D. W. Wichern, Applied multivariate statistical analysis. Prentice hall Englewood Cliffs, NJ, 1992, vol. 4.

[13] "Generalized variance - multivariate normal distribution," [Online] Available: http://jonathantemplin.com/files/multivariate/mv05psyc990/ psyc990_04.pdf, 2005.

[14] M. D. McKay, R. J. Beckman, and W. J. Conover, "Comparison of three methods for selecting values of input variables in the analysis of output from a computer code," Technometrics, vol. 21, no. 2, pp. 239-245, 1979.

[15] M. Stein, "Large sample properties of simulations using latin hypercube sampling," Technometrics, vol. 29, no. 2, pp. 143-151, 1987.

[16] D. S. Moore and G. P. McCabe, Introduction to the Practice of Statistics, 6 th ed. New York Freeman, 2009.

[17] E. Heylen, G. Deconinck, and D. Van Hertem, "Impact of value of lost load on performance of power system reliability criteria and their management," in Proc. 2015 IEEE PowerTech, Jun. 2015.

[18] R. Billinton, S. Kumar, N. Chowdhury, K. Chu, K. Debnath, L. Goel, E. Khan, P. Kos, G. Nourbakhsh, and J. Oteng-Adjei, "A reliability test system for educational purposes-basic data," IEEE Trans. Power Syst., vol. 4, no. 3, pp. 1238-1244, Aug. 1989.

[19] R. D. Zimmerman, C. E. Murillo-Sánchez, and R. J. Thomas, "Matpower: Steady-state operations, planning, and analysis tools for power systems research and education," IEEE Trans. Power Syst., vol. 26, no. 1, pp. 12-19, Feb. 2011 